\title{
Regards croisés sur un patrimoine textile inédit
}

Made in Neuchâtel. Deux siècles d'indiennes, Musée d'art et d'histoire de Neuchâtel, 7 octobre 2018-19 mai 2019

\section{Lisa Laurenti}

\section{(2) OpenEdition}

1 Journals

\section{Édition électronique}

URL : http://journals.openedition.org/artefact/4788

DOI : 10.4000/artefact.4788

ISSN : 2606-9245

Éditeur :

Association Artefact. Techniques histoire et sciences humaines, Presses universitaires du Midi

\section{Édition imprimée}

Date de publication : 15 décembre 2019

Pagination : 197-200

ISBN : 978-2-8107-0667-9

ISSN : 2273-0753

Référence électronique

Lisa Laurenti, «Regards croisés sur un patrimoine textile inédit », Artefact [En ligne], 11 | 2019, mis en ligne le 27 novembre 2020, consulté le 29 novembre 2020. URL : http://journals.openedition.org/ artefact/4788; DOI : https://doi.org/10.4000/artefact.4788

\section{(c) (i) (9)}

Artefact, Techniques, histoire et sciences humaines est mise à disposition selon les termes de la Licence Creative Commons Attribution - Pas d'Utilisation Commerciale - Pas de Modification 4.0 International. 


\title{
Regards croisés sur un patrimoine textile inédit
}

\author{
Made in Neuchâtel. Deux siècles d'indiennes, Musée d'art \\ et d'histoire de Neuchâtel, 7 octobre 2018-19 mai 2019
}

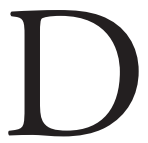

u 7 octobre 2018 au 19 mai 2019, le Musée d'art et d'histoire de Neuchâtel a présenté une grande exposition intitulée « Made in Neuchâtel. Deux siècles d'indiennes ", à partir des riches collections du Musée et de prêts internationaux. Il s'agissait de la première exposition temporaire que l'institution dédiait aux indiennes produites à Neuchâtel aux XVIII ${ }^{e}$ et $\mathrm{XIX}^{\mathrm{e}}$ siècles. Privilégiant une approche interdisciplinaire, le parcours interrogeait les stratégies mises en œuvre par les producteurs et les négociants pour conquérir de vastes marchés au niveau mondial, tout en éclairant les raisons géographiques, politiques et économiques qui ont favorisé l'implantation, le développement et le déclin de cette florissante industrie dans la région. Une publication, richement illustrée, coéditée à cette occasion avec Somogy Éditions d'art, a réuni des contributions de spécialistes, suisses et internationaux, qui éclairent les principaux aspects traités dans l'exposition. Des notices détaillées des objets les plus significatifs des collections complètent ces présentations.

Les indiennes ont suscité un engouement sans précédent en Europe aux $\mathrm{XVIII}^{\mathrm{e}}$ et $\mathrm{XIX}^{\mathrm{e}}$ siècles. Neuchâtel se plaça très rapidement parmi les principaux centres de production d'indiennes sur le continent. À la fin du $\mathrm{XVIII}^{\mathrm{e}}$ siècle, une quinzaine de manufactures installées dans la région occupait jusqu'à 2000 personnes, hommes, femmes et enfants, soit environ un dixième de la population active de la Principauté. Près de deux millions de toiles destinées avant tout à l'exportation y sont fabriquées annuellement.

95 Lisa Laurenti, « Regards croisés sur un patrimoine textile inédit », Artefact, 11, 2019, p. 197-200. 
Producteurs et négociants ont développé des stratégies très diverses pour conquérir les marchés internationaux. Ils ont mis en place des réseaux commerciaux en Europe mais également en Asie, en Afrique et dans les colonies d'Amérique, au travers desquels ils se fournissaient en toiles blanches et en produits tinctoriaux. Pour l'écoulement des toiles imprimées, ils participaient régulièrement aux principales foires européennes et bénéficiaient d'un réseau d'intermédiaires qui leur ont permis d'adapter sans cesse le langage ornemental des indiennes « made in Neuchâtel » aux tendances en vogue et de répondre ainsi à une clientèle diversifiée issue de plusieurs aires géographiques, socio-économiques et culturelles.

Le projet était le résultat du travail d'une équipe pluridisciplinaire. Le commissariat a été assuré par Lisa Laurenti ${ }^{1}$ et Philippe Lüscher, conservatrice-assistante et conservateur des collections d'arts appliqués du Musée d'art et d'histoire de Neuchâtel. Comme le suggérait le titre de l'exposition, l'indiennage a été appréhendé à travers le filtre neuchâtelois, une approche qui a permis de porter un nouveau regard sur la création, la production et la commercialisation des indiennes dans la perspective de la mondialisation des XVIII ${ }^{e}$ et XIX ${ }^{e}$ siècles. À partir d'un corpus inédit de toiles, de sources et d'objets, l'exposition entendait montrer ce qu'est une indienne produite dans la région. Histoire du textile et de la mode, histoire économique et culturelle s'articulaient et se complétaient tout au long de l'exposition. La création et la circulation du langage ornemental, les stratégies de production et de vente, ainsi que les techniques d'impression étaient présentées dans un discours à la fois local et global. Le contexte historique - les liens de Neuchâtel avec la Prusse, l'apport de réfugiés huguenots dans l'essor de l'indiennage de la région et le rôle de Neuchâtelois dans la traite des Noirs et l'esclavage - était mis en évidence.

$\mathrm{Au}$ cour du parcours muséographique le public pouvait découvrir de riches ensembles d'objets représentatifs du goût et de l'esthétique des XVIII ${ }^{\mathrm{e}}$ et $\mathrm{XIX}^{\mathrm{e}}$ siècles qui, par comparaison, permettaient de dessiner la géographie des relations commerciales, des influences culturelles et des circulations de savoirs et de personnes. Le foisonnement d'artefacts, minutieusement étudiés, datés et attribués, trouvait une place incontournable à l'intérieur des différentes sections développées. Chaque objet éclairait par sa matérialité et

1. Lisa Laurenti prépare une thèse de doctorat en histoire de l'art sur les indiennes à l'Université de Lausanne sous la direction du Prof. Dave Lüthi. 
sa signification deux siècles d'indiennage à Neuchâtel (fig. VIII-X, cahier couleur).

Face à la profusion, aujourd'hui, de motifs inspirés des indiennes dans la haute couture et le prêt-à-porter, les commissaires ont invité des spécialistes, des créateurs et des artisans à travailler avec des objets phares des collections d'indiennes, afin d'offrir un questionnement d'actualité sur ce domaine spécifique des arts manufacturés. Leur regard contemporain a permis de tisser un lien entre passé et présent, tout en rythmant et dynamisant l'expérience du visiteur.

Ce dernier était d'ailleurs d'emblée confronté, dès l'entrée de l'exposition (fig. VII, cahier couleur), aux créations contemporaines des étudiant-e-s de la filière Design Textile de la Haute École spécialisée de Lucerne avec laquelle le Musée a mis en ouvre un partenariat. La flore, qui a fasciné le $\mathrm{XVIII}^{\mathrm{e}}$ siècle, ou le motif cachemire, qui a tant marqué la mode vestimentaire du XIX ${ }^{e}$ siècle, étaient ainsi interprétés et déclinés dans une multitude de tons et de compositions par des créateurs habiles à jongler entre tradition, création et exigences industrielles. Comme par le passé, l'interdépendance entre création de modèles, commercialisation et consommation fait partie des processus de conception des designers textiles du XXI ${ }^{e}$ siècle (fig. XI, cahier couleur).

L'exposition a été l'occasion de développer un autre projet réunissant des spécialistes suisses et indiens - notamment le Talianna Studio à Delhi afin de montrer les liens entre les techniques d'impression pratiquées en Inde hier et aujourd'hui. Cette collaboration a permis de mettre en évidence en particulier les technologies employées actuellement dans l'industrie et le design.

Dans le domaine de la conservation, un partenariat inédit a été conclu avec la Fondation Bill de Vigier, qui nous a conduit à présenter la restauration d'une rare chambre à alcôve tapissée en indiennes de la fin du XVIII ${ }^{\mathrm{e}}$ siècle, qui se trouve à la Sommerhaus de Vigier à Soleure. L'opération a été menée en 2017-2018 par ladite fondation. Sa présentation dans l'exposition a permis d'aborder les problématiques contemporaines d'attribution, de datation et de restauration-conservation du domaine textile.

L'exposition porte une attention particulière à la médiation. Tout au long du parcours, des animations audiovisuelles et une installation interactive 
offrent au visiteur la possibilité de découvrir de nouvelles dimensions des objets. Un riche programme-cadre d'intérêt scientifique et culturel a été mis en place. Ces événements ont permis, non seulement de proposer des activités pédagogiques et des visites guidées autour des nombreux aspects traités dans l'exposition, mais également de présenter les acquis de la recherche dans le domaine. Une table ronde et une conférence réunissant des historien-ne-s suisses, français-es et américain-e-s ont été consacrées à la question de la traite des Noirs et de l'esclavage en lien avec l'indiennage. Les sources de la création et du langage ornemental des indiennes, dans la perspective des arts décoratifs, sont autant de sujets nouvellement traités dans la recherche académique : richement présentés dans les salles de l'exposition, ils ont fait pareillement l'objet d'une conférence.

L'intérêt que l'exposition a suscité, tant auprès des spécialistes que d'un large public suisse et international, témoigne également de l'actualité du sujet et de son importance pour l'histoire culturelle, artistique et économique. Le Musée d'art et d'histoire de Neuchâtel a verni son exposition quelques mois après l'inauguration au Musée national suisse - Château de Prangins - de l'exposition "Indiennes. Un tissu révolutionne le monde ! » (22 avril-14 octobre 2018) qui retraçait l'histoire des indiennes, de leurs origines en Inde à leur fabrication en Europe, en interrogeant en particulier la contribution des Suisses à cette industrie. 